\title{
High-dose chemotherapy and autologous stem cell rescue for atypical teratoid/rhabdoid tumor of the central nervous system
}

\author{
Theodore Nicolaides $\cdot$ Tarik Tihan $\cdot$ Biljana Horn $\cdot$ \\ Jaclyn Biegel $\cdot$ Michael Prados $\cdot$ Anuradha Banerjee
}

Received: 22 September 2009/ Accepted: 9 November 2009/Published online: 22 November 2009

(C) The Author(s) 2009. This article is published with open access at Springerlink.com

\begin{abstract}
Atypical Teratoid/Rhabdoid tumors (AT/RT) of the central nervous system are rare but aggressive tumors of childhood. Median survival with surgery and standard chemotherapy is less than 12 months. In an attempt to improve outcome, patients were treated with aggressive surgical resection and multi-agent chemotherapy, followed by high dose chemotherapy with autologous stem cell rescue. Nine consecutive children (median age 21 months) were diagnosed with AT/RT at the University of California San Francisco Childrens Hospital from 1997 to 2007 and treated with this aggressive approach. Diagnosis was confirmed using molecular markers. There are two long-term survivors (78 and 98 months from diagnosis). One additional patient is alive with disease. Three patients died of disease during therapy. Three patients died of disease after therapy was complete. There were no toxic deaths. Two of nine patients treated for AT/RT at our institution with high
\end{abstract}

T. Nicolaides $(\bowtie) \cdot$ B. Horn · A. Banerjee

Department of Pediatrics, University of California San Francisco

School of Medicine, 505 Parnassus Avenue, M649, Box 0106,

San Francisco, CA 94143, USA

e-mail: Theodore.Nicolaides@ucsf.edu

\section{T. Tihan}

Department of Pathology, University of California San Francisco School of Medicine, 505 Parnassus Avenue, M551C, Box 0102, San Francisco, CA 94143, USA

\section{T. Tihan}

UCSF Children's Hospital, University of California San

Francisco School of Medicine, 505 Parnassus Avenue, M551C,

Box 0102, San Francisco, CA 94143, USA

T. Nicolaides $\cdot$ B. Horn $\cdot$ A. Banerjee

UCSF Children's Hospital, University of California San

Francisco School of Medicine, 505 Parnassus Avenue, M649,

Box 0106, San Francisco, CA 94143, USA dose chemotherapy and autologous bone marrow transplant are long-term survivors, suggesting that a subset of patients can be cured with this approach.

Keywords CNS tumors .

Atypical teratoid/rhabdoid tumor - Autologous transplant

\section{Introduction}

Malignant rhabdoid tumors (MRT) are a rare group of aggressive childhood tumors that most often arise in the kidney. First described in 1978, these tumors have characteristic large polygonal cells that contain globular, eosinophilic cytoplasm and have vesicular nuclei, often with prominent nucleoli [1]. These cells were reminiscent of rhabdomyoblasts and so were called rhabdoid cells. Reports

\author{
J. Biegel \\ Children's Hospital of Philadelphia, University of Pennsylvania, \\ 3615 Civic Center Boulevard, Room 102, Philadelphia, \\ PA 19104, USA \\ M. Prados \\ Departments of Neurosurgery, University of California San \\ Francisco School of Medicine, 400 Parnassus Avenue, Box 0372, \\ San Francisco, CA 94143, USA \\ M. Prados \\ UCSF Children's Hospital, University of California San \\ Francisco School of Medicine, 400 Parnassus Avenue, Box 0372, \\ San Francisco, CA 94143, USA
}


of primary intracranial tumors containing rhabdoid cells exist as early as 1985 [2]. These heterogeneous tumors affect young children and are composed of neuroepithelial, peripheral epithelial, and mesenchymal elements (in addition to their characteristic rhabdoid cells) and were later coined atypical teratoid/rhabdoid tumors (AT/RT) by Rorke and others in 1995 [3]. Sections of these tumors can appear identical to primitive neuro-ectodermal tumors (PNET's) or germ cell tumors on histology and therefore require immunohistochemical and cytogenetic data for definitive diagnosis.

Many of these tumors were classified under the generic category of PNET in the past. However, the exact histologic characterization is crucial as treatment outcomes may differ. PNET/Medulloblastoma (MB)'s, for example, can respond well to aggressive surgery and chemotherapy alone [4]. AT/RT patients, on the other hand, have a very poor prognosis when treated on PNET-MB protocols [5] and respond only marginally better when treated with more aggressive therapy. To date, approximately 200 cases of AT/RT have been reported in the literature.

In this publication, we report on nine children identified at our institution. All patients had their INI1 status examined at the protein or gene level and only those patients found to be altered were included for further analysis. Histological and molecular features as well as treatment outcome are reported.

\section{Methods}

\section{Inclusion criteria}

All patients with newly diagnosed with central nervous system AT/RT between May 1997 and January 2007 at the University of California San Francisco (UCSF) are described. Patient characteristics are shown in Table 1. This study was approved by our institutional Committee on Human Research.

The diagnosis of CNS AT/RT was re-confirmed by one of the authors (T.T) for all patients. The pathological findings are presented in Table 2 and include molecular diagnostics (immunohistochemistry for BAF47 or mutational analysis for INI1) for all patients. BAF47 immunohistochemistry was performed using the BAF47/SNF5 mouse monoclonal antibody (BD Transduction Labs, San Diego, CA) and INI1 mutation analysis was performed as previously described [6]. Representative histology and immunohistochemistry are shown in Fig. 1.

\section{Results}

Clinical features are presented in Table 1. All patients presented with signs of increased intracranial pressure. Of our nine patients, seven were male, which is in agreement with the 2:1 male:female ratio reported by the ATRT Registry [7]. The median age of diagnosis was 21 months (range 6-49 months). Four out of nine tumors arose in a twin. The tumors arose from throughout the neuraxis, and disseminated disease was common. Six out of the nine patients underwent a gross total resection (GTR), while three had either a subtotal resection (STR) or a biopsy.

Adjuvant chemotherapy regimens included cisplatin, vincristine, cyclophosphamide, and etoposide (used in Childrens Cancer Group study 99703) or high-dose methotrexate, cisplatin, vincristine, cytoxan, and etoposide (used in the HeadStart-II study) [8]. Only one patient received radiation therapy (Patient 2 received 64 Gray conformal radiation prior to transplant).

Six patients achieved or sustained a complete response (CR) with induction chemotherapy, while three patients progressed during induction chemotherapy. Patients that achieved a CR then went onto high dose chemotherapy with autologous stem cell transplant (ASCT). Transplant characteristics are described in Table 1. Transplants were well tolerated and all patients engrafted within 16 days.

Follow-up duration ranged from 6 to 98 months. Median Progression-Free Survival (PFS) as well as overall survival (OS) was 10 months (range 1-98 months) (Fig. 2). There were two long term survivors (PFS of 98 and 78 months). Both of these patients underwent ASCT.

\section{Discussion}

Since being recognized as an independent entity, it has become clear that patients with AT/RT have a dismal prognosis. The original series described a median survival of 6 months [3]. Subsequent treatment strategies have consisted of intensive multi-modal therapy with surgery, chemotherapy and/or radiation therapy. Because of the rarity of the disease, clinical reports of patients with AT/RT have been mostly anecdotal.

In this paper, we describe the series of patients treated for AT/RT at our institution. As with other malignant pediatric brain tumors, gross total resection (GTR) seemed to be associated with better outcome in our series, with both long-term survivors having had GTR's, though the small numbers precluded statistical evaluation (Fig. 2). This is in agreement with the largest reported study of AT/ RT patients thus far, based on the multi-center national registry. Retrospective data compiled on the AT/RT registry [7] reported OS of 20 months in 20 patients with GTR versus 15 months in 22 patients with sub-total resection or biopsy. Additionally, a recent report from Chi et al. [9] demonstrated the importance of GTR in a prospective multi-center trial. In that study, both overall survival and 


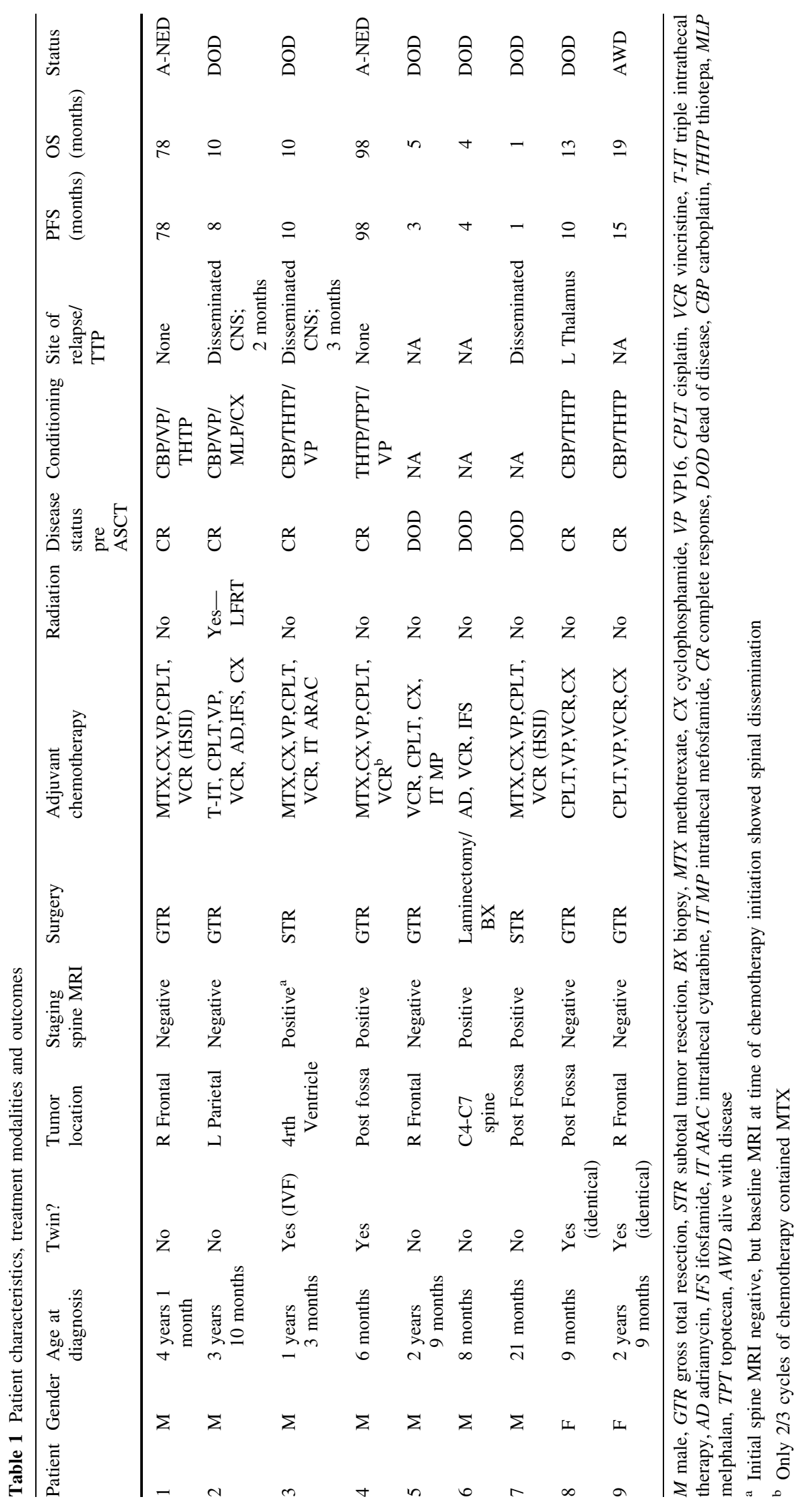


Table 2 Pathological characteristics

\begin{tabular}{|c|c|c|c|c|c|c|c|c|}
\hline Patient & Rhabdoid cells & EMA & Vimentin & S100 & GFAP & Synaptophysin & BAF47 Nuclear staining & Mutation analysis \\
\hline 1 & + & + & + & - & \pm & - & Absent & + \\
\hline 2 & + & & + & + & - & - & & + \\
\hline 3 & + & + & + & + & & + & & + \\
\hline 4 & + & + & + & + & - & + & Absent & + \\
\hline 5 & + & - & + & & & - & Absent & + \\
\hline 6 & + & - & + & - & & - & Absent & Not Done \\
\hline 7 & + & + & + & + & - & + & Absent & Not Done \\
\hline 8 & + & + & + & & + & & Absent & Not Done \\
\hline 9 & + & - & + & - & - & + & Absent & Not Done \\
\hline
\end{tabular}
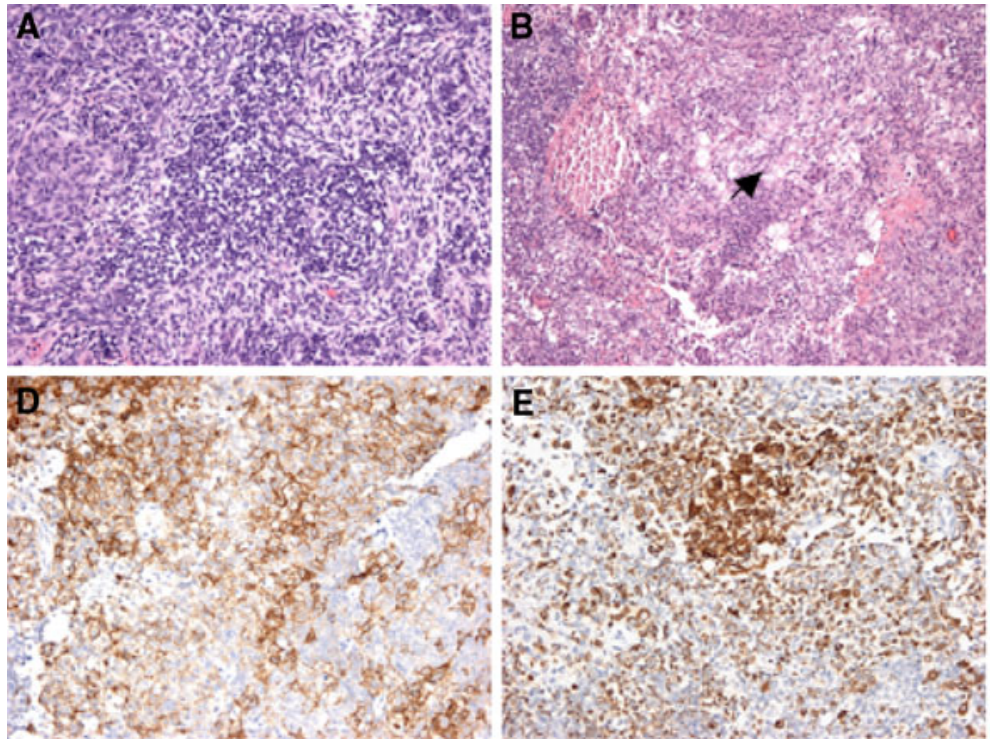
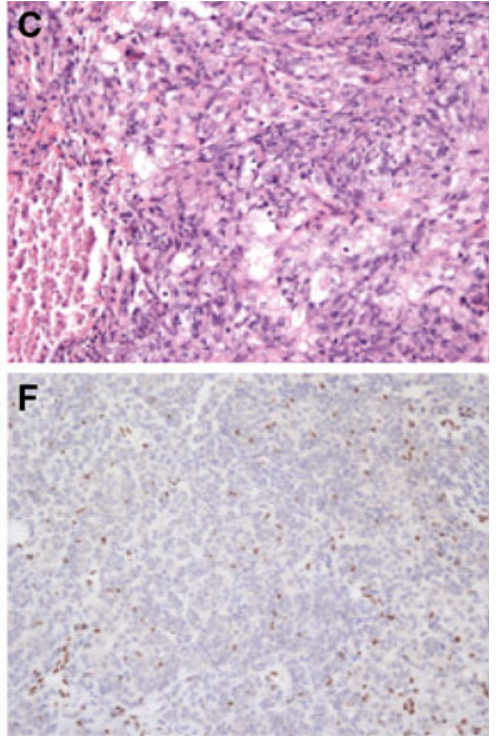

Fig. 1 Immuno-histochemistry. a $\mathrm{H}$ and $\mathrm{E}$ of small cell component of AT/RT. b H and E of large cell component, with necrotic area (arrow). c Higher magnification of b. d Positive EMA stain. e Positive

progression free survival were significantly higher in the group of patients that underwent GTR. Unfortunately, GTR is often not possible due to disseminated disease at diagnosis or disease located adjacent to vital structures. Adjuvant therapy is required to cure patients with AT/RT, but identifying the best therapy has proven difficult.

The role for radiation therapy is controversial in this disease (reviewed in [10]). Although the incorporation of external beam radiation therapy has resulted in some long term survivors in children greater than 3 years of age [11], most clinicians are hesitant to irradiate "infants" or children younger than 3 years of age due to the significant risk of neuro-endocrine toxicity (reviewed in [12]). In order to avoid irradiation (or at least delay it until after 3 years of age), clinicians have used high dose chemotherapy with autologous stem cell transplantation (HD-ASCT). This
Cytokeratin stain. f BAF47/INI1 antibody stain showing loss of INI1 expression in AT/RT. Retained expression is noted in scattered inflammatory cells, constituting a positive internal control

approach has increased survival in young children with recurrent MB's and supratentorial PNET's [13, 14].

Adjuvant chemotherapy used to treat AT/RT's is aggressive and published series in the literature have used one of two chemotherapeutic backbones: Intergroup Rhabdomyosarcoma III (IRS-III) and Children's Cancer Group (CCG)-9921. Weinblatt and Kochen [15] first reported the successful use of surgery, radiation therapy, and combination chemotherapy using the IRS-III regimen 36 (originally designed to treat parameningeal rhabdomyosarcoma) to treat a child with AT/RT. This chemotherapeutic regimen consists of vincristine, cisplatin, doxorubicin, cyclophosphamide, dacarbazine (replaced by the related drug temozolomide in modern protocol), etoposide, actinomycin-D and triple intrathecal therapy with methotrexate, hydrocortisone, and cytarabine. Olsen et al. [16] reported 

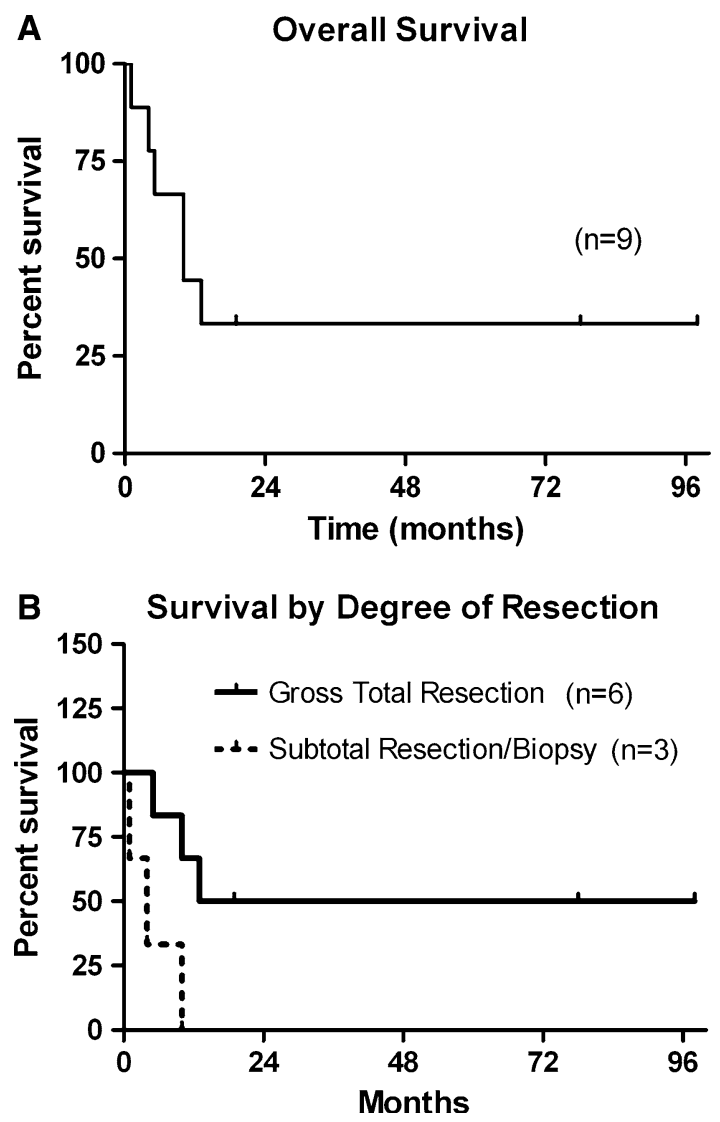

Fig. 2 Survival curves. a Kaplan-Meier curve showing overall survival. b Kaplan-Meier curve showing survival based on extent of resection

on an additional two long term survivors using this approach, while Zimmerman et al. [17] successfully treated four consecutive patients using this approach. Of particular note, two of the patients from the latter report were relapsed patients who had failed conventional therapy. This regimen can clearly be effective, but the requirement for radiation therapy (focal stereotactic radiation therapy in "infants") is controversial. One of the three "infants" treated by Zimmerman et al. is reported to have significant cognitive delay. During the preparation of this manuscript, the results of a prospective multi-center trial using aggressive surgery, IRS-III based induction chemotherapy (including intrathecal chemotherapy) and radiation therapy to all patients (including patients under 3 years of age) was published. Chi et al. [9] reported 2-year progression-free and overall survival rates of 53 and $70 \%$, respectively, in a cohort of 20 patients. This report is extremely encouraging and there will be much interest in the long-term outcome of this cohort.

CCG-9921 (Regimen A) based therapy uses a four drug induction regimen (vincristine, cisplatin, cyclophosphamide, etoposide) found to be the most active arm against embryonal tumors [18]. This backbone has been used as the induction regimen for the "Headstart" protocols and for CCG-99703 which add HD-ASCT in place of radiation therapy in order to further intensify therapy in "infants". A recent report by Gardner et al. [8] described the outcomes of AT/RT patients treated with the Headstart protocols. The prospective study spanned 10 years and two versions of the protocol, with "Headstart II" adding high dose methotrexate to the regimen. Myeloablative chemotherapy prior to transplant consisted of carboplatin, thiotepa and etoposide. While high dose methotrexate has activity against embryonal brain tumors [19], it also significantly adds to the rate of toxic deaths. The prospective report from Gardner et al. describes thirteen patients treated on Headstart I and II and reports three long term survivors. Two out of three of these patients received induction with high dose methotrexate. Also, most patients in Gardner's study did not have confirmation with INI1 analysis, as they were treated before the test became available. However, the three long term survivors have had their diagnosis confirmed by INI1 analysis (S.L. Gardner, personal communication).

Our study reports on nine consecutive patients treated at a single institution for AT/RT. Induction chemotherapy varied in our series. Four patients were treated per Headstart II, but only one of these patients was treated on study. That patient (\#4 in our study) is a long term survivor and was previously reported (as patient HSII \#6) in the recent report by Gardner et al. [8]. Two patients were treated per CCG-99703 and the others varied. Although induction chemotherapy varied in our series, all patients were treated with intensive chemotherapy and then taken to HD-ASCT if they were in CR. Myeloablative chemotherapy consisted of one cycle for four patients, and of three smaller transplants for the two patients on CCG-99703. Our patients tolerated therapy well with no toxic deaths. Of the six patients that underwent HD-ASCT, three remain alive at the time of this report, with two of these patients being long term survivors ( $>5$ years survival).

All patients in our study had their diagnosis confirmed with the histological presence of rhabdoid cells, immunohistochemical evidence of epithelial and/or mesenchymal markers and the inactivation of INI1 - defined by either INI1 mutation/deletion or loss of nuclear expression of BAF47. Confirmation of an AT/RT diagnosis is critical as clinical trials are being designed for AT/RT. In a recent review of INI1 staining in CNS tumors, Judkins [20] reported that all 68 reported cases of AT/RT's with INI1 gene alterations have been found to lose expression of INI1 on immunohistochemistry. Additionally, the only three reported cases of CNS tumors with loss of expression of INI1 that were not AT/RT's were two oligodendrogliomas and a pituitary adenoma, which were readily differentiated from AT/RT based on their histological appearance. 
The key role of INI1 in the pathogenesis of AT/RT's has led investigators to attempt to target this pathway. The current paradigm of targeted therapy for cancer has focused on the use of small molecule kinase inhibitors or monoclonal antibodies that inactivate pathways with gain of function abnormalities. The fact that AT/RT's lose the function of INI1 makes targeted therapy in this disease a difficult task. Recent evidence suggests, however, that the Insulin-like growth factor 1 receptor (IGF-1R) and one of its ligands, IGF-II, form an autocrine/paracrine loop in AT/RT's and that this may be crucial to tumorigenesis [21]. Targeting the IGF-1R with antisense oligonucleotides or a small molecule inhibitor to IGF-1R has shown efficacy in vitro $[22,23]$. Monoclonal antibodies targeting IGF-1R are currently having some success in clinical trials for sarcomas, while small molecule inhibitors of IGF-1R are still being investigated [24]. This suggests that targeted therapy for AT/RT's may one day be possible.

One additional interesting observation from our series was the high incidence of patients who were from twin pregnancies (4/9 or $44 \%$ ), which is significantly higher (3/100 being the incidence of twins in California in 2005) than expected by chance (Fisher's exact test; $P<0.05$ ). One of these patients was the product of in vitro fertilization, which itself has been associated with infant embryonal malignancies [25]. Although there is a prior report of a twin with AT/RT [26], the small numbers involved make it difficult to determine if an increased risk is truly associated with multiple gestations. Future studies on AT/RT should attempt to collect the information on presence of multiple gestations.

In summary, young patients with AT/RT continue to have a poor prognosis. Adding to previously published reports, we confirm that only a small proportion of patients with AT/RT can be cured with high dose chemotherapy and autologous stem cell rescue. However, identifying patients who will favorably respond to this therapy remains difficult. Although gross total resection is clearly an important factor in improving survival rates, the high rate of treatment failure in the group of totally resected patients suggests that there might be additional factors that can modify the long-term response to chemotherapy. The recent report from Chi et al. [9] suggests that radiation therapy, when coupled with IRS-III based chemotherapy, may be an important treatment strategy in this disease. Given the small number of patients with AT/RT, a large multi-institutional trial is needed to answer the many therapeutic questions in this disease. Encouragingly, the Childrens Oncology Group has recently initiated a therapeutic protocol specifically for AT/RT patients. This protocol provides Headstart-II based induction chemotherapy, followed by conformal radiation, and high dose chemotherapy with autologous stem cell rescue. Lastly, new agents are required for treatment of this rare but deadly disease. Preclinical data has identified the IGF-1R pathway as a potential target in this disease.

Acknowledgements This study was supported by the Campini Foundation (T.N. \& A.B.), American Brain Tumor Association (T.N.), NIH T32 CA108462-01 (T.N.) and NIH CA46274 (J.B.). Thanks to Kate Matthay for critical review of this manuscript.

Open Access This article is distributed under the terms of the Creative Commons Attribution Noncommercial License which permits any noncommercial use, distribution, and reproduction in any medium, provided the original author(s) and source are credited.

\section{References}

1. Beckwith JB, Palmer NF (1978) Histopathology and prognosis of Wilms tumors: results from the First National Wilms' Tumor Study. Cancer 41:1937-1948

2. Briner J, Bannwart F, Kleihues P et al (1985) Malignant small cell tumor of the brain with intermediate filaments - a case of a primary cerebral rhabdoid tumor. Pediatr Pathol 3:117-118

3. Rorke LB, Packer R, Biegel J (1995) Central nervous system atypical teratoid/rhabdoid tumors of infancy and childhood. J Neurooncol 24:21-28

4. Rutkowski S, Bode U, Deinlein F et al (2005) Treatment of early childhood medulloblastoma by postoperative chemotherapy alone. N Engl J Med 352:978-986

5. Burger PC, Yu IT, Tihan T et al (1998) Atypical teratoid/rhabdoid tumor of the central nervous system: a highly malignant tumor of infancy and childhood frequently mistaken for medulloblastoma: a Pediatric Oncology Group study. Am J Surg Pathol 22:1083-1092

6. Judkins AR, Mauger J, Ht A et al (2004) Immunohistochemical analysis of hSNF5/INI1 in pediatric CNS neoplasms. Am J Surg Pathol 28:644-650

7. Hilden JM, Meerbaum S, Burger P et al (2004) Central nervous system atypical teratoid/rhabdoid tumor: results of therapy in children enrolled in a registry. J Clin Oncol 222:2877-2884

8. Gardner SL, Asgharzadeh S, Green A et al (2008) Intensive induction chemotherapy followed by high dose chemotherapy with autologous hematopoietic progenitor cell rescue in young children newly diagnosed with central nervous system atypical teratoid rhabdoid tumors. Pediatr Blood Cancer 51:235-240

9. Chi SN, Zimmerman MA, Yao X et al (2009) Intensive multimodality treatment for children with newly diagnosed CNS atypical teratoid rhabdoid tumor. J Clin Oncol 27(3):385-389

10. Squire SE, Chan MD, Marcus KJ (2007) Atypical teratoid/rhabdoid tumor: the controversy behind radiation therapy. J Neurooncol 81:97-111

11. Tekautz TM, Fuller CE, Blaney S et al (2005) Atypical teratoid/ rhabdoid tumors (ATRT): improved survival in children 3 years of age and older with radiation therapy and high-dose alkylatorbased chemotherapy. J Clin Oncol 23:1491-1499

12. Duffner PK (2004) Long-term effects of radiation therapy on cognitive and endocrine function in children with leukemia and brain tumors. Neurologist 10:293-310

13. Dunkel IJ, Boyett JM, Yates A et al (1998) High-dose carboplatin, thiotepa, and etoposide with autologous stem-cell rescue for patients with recurrent medulloblastoma. Children's Cancer Group. J Clin Oncol 16:222-228

14. Broniscer A, Nicolaides TP, Dunkel IJ et al (2004) High-dose chemotherapy with autologous stem-cell rescue in the treatment 
of patients with recurrent non-cerebellar primitive neuroectodermal tumors. Pediatr Blood Cancer 42:261-267

15. Weinblatt M, Kochen J (1992) Rhabdoid tumor of the central nervous system. Med Pediatr Oncol 20(3):258

16. Olson TA, Bayar E, Kosnik E et al (1995) Successful treatment of disseminated central nervous system malignant rhabdoid tumor. J Pediatr Hematol Oncol 17:71-75

17. Zimmerman MA, Goumnerova LC, Proctor M et al (2005) Continuous remission of newly diagnosed and relapsed central nervous system atypical teratoid/rhabdoid tumor. J Neurooncol 72:77-84

18. Geyer JR, Sposto R, Jennings M et al (2005) Multiagent chemotherapy and deferred radiotherapy in infants with malignant brain tumors: a report from the Children's Cancer Group. J Clin Oncol 23:7621-7631

19. Allen JC, Walker R, Rosen G (1988) Preradiation high-dose intravenous methotrexate with leucovorin rescue for untreated primary childhood brain tumors. J Clin Oncol 6:649-653

20. Judkins AR (2007) Immunohistochemistry of INI1 expression: a new tool for old challenges in CNS and soft tissue pathology. Adv Anat Pathol 14(5):335-339
21. Ogino S, Cohen ML, Abdul-Karim FW (1999) Atypical teratoid/ rhabdoid tumor of the CNS: cytopathology and immunohistochemistry of insulin-like growth factor-II, insulin-like growth factor receptor type 1, cathepsin D, and Ki-67. Mod Pathol 12(4):379-385

22. D'cunja J, Shalaby T, Rivera P et al (2007) Antisense treatment of IGF-IR induces apoptosis and enhances chemosensitivity in central nervous system atypical teratoid/rhabdoid tumours cells. Eur J Cancer 43:1581-1589

23. Arcaro A, Doepfner KT, Boller D et al (2007) Novel role for insulin as an autocrine growth factor for malignant brain tumour cells. Biochem J 406:57-66

24. Pollak M (2008) Insulin and insulin-like growth factor signalling in neoplasia. Nat Rev Cancer 8:915-928

25. Toren A, Sharon N, Mandel M et al (1995) Two embryonal cancers after in vitro fertilization. Cancer 76(11):2372-2374

26. Fernandez C, Bouvier C, Sevenet N et al (2002) Congenital disseminated malignant rhabdoid tumor and cerebellar tumor mimicking medulloblastoma in monozygotic twins: pathologic and molecular diagnosis. Am J Surg Pathol 26:266-270 